\title{
Comparison of Dried Blood Spot Collection Techniques in Nigeria
}

\author{
George I. Eluwa ${ }^{1 *}$, Adebobola Bashorun ${ }^{2}$, Emmanuel Abata ${ }^{2}$, Ogboi S. Johnbull ${ }^{3}$ \\ ${ }^{1}$ Marie Stopes International Organization, Nigeria \\ ${ }^{2}$ Division of HIV/AIDS, Federal Ministry of Health, Nigeria \\ 3 Jedima International Health Consult Ltd, Lagos, Nigeria
}

Received: March 01, 2016; Accepted: July 10, 2016; Published: July 15, 2016

*Corresponding author: George I. Eluwa, Marie Stopes International Organisation, Nigeria. 59 Anthony Enahoro Street, Utako, Abuja, Nigeria, Tel: +2347081403744; E-mail: dreluwag@gmail.com

\begin{abstract}
Absract
Background: Studies have focused on the type of filter paper to be used in sample collection for national surveys with optimum care in minimizing errors in field implementation among health personnel. Therefore, the aim of this study was to compare the HIV antigen/ antibody results of different dried blood spot collection devices.

Methods: Dried blood spots were collected from key populations on both conventional DBS cards and HemaSpot ${ }^{\mathrm{TM}}$ which both used Munktell TFN (M-TFN) filter paper. Samples from the DBS cards were tested on HIV ELISA analysis using Vironostika 4th Generation ELISA assay. Bland-Altman analysis was used to measure limit level of agreement.
\end{abstract}

Results: The overall sero-positivity of HIV rapid test, conventional DBS card and HemaSpot ${ }^{\mathrm{TM}}$ was $7 \%, 67 \%$ and $29 \%$ respectively. Of the 96 samples accepted and tested with the assay, concordance between conventional DBS cards and HemaSpot $^{\mathrm{TM}}$ was $46.9 \%$ (n = 45). Concordance between rapid test and conventional DBS card was $39.5 \%(\mathrm{n}=34)$ while between rapid test and HemaSpot $^{\mathrm{TM}}$ was $75.6 \%$ $(\mathrm{n}=65)$. Bland-Altman analysis showed poor agreement between both DBS collection devices with a mean of 0.149 and a standard deviation of 0.641 , which yields a limit of agreement of -0.717 to 1.847 .

Conclusion: Concordance with rapid test was higher with HemaSpot $^{\mathrm{TM}}(76 \%)$ compared to conventional DBS card $(40 \%)$. As both devices used the same filter paper, it is unlikely that the increase in sero-positivity in conventional DBS card and HemaSpot ${ }^{\mathrm{TM}}$ is exclusive to new infections identified by the antigenic capability of the ELISA kit. However, the high level of discordance of results, especially between rapid test and conventional DBS card calls for further research and necessitates a more rigorous quality control in sample collection.

\section{Introduction}

The World Health Organization provides guidelines for monitoring the development and transmission of HIV infections among general population, key target population, mother to child transmission and HIV drug resistance [1,2]. In response to the Federal Ministry of Health in Nigeria set up a surveillance system to determine the magnitude of the HIV problem, as well as monitor trends of the epidemic. The surveillance involved prevalence rates for HIV among various groups such as women attending antenatal clinics, blood donors, transport workers and patients attending sexually transmitted infection (STI) clinics in the country. Such surveys are essential for providing evidence of the impact of HIV interventions, tracking trends of HIV prevalence and maintaining the efficacy of antiretroviral drug regimens within the population in areas where individualized patient monitoring is not available. A key aspect of these surveillance activities is the collection of bio-repository samples for use in secondary analysis as a means of quality control.

The gold standard of specimen type for most surveys is plasma, however due to the increased cost and logistical challenges of separating and maintaining frozen plasma specimens, several studies investigated alternative specimen types for HIV antibody and antigen analysis [1]. In resourceconstrained settings with poor logistics and weak health system, alternate methods of specimen collection that do not require cold chain increase the efficiency of the health system as cost of sample transfer is reduced as well increasing the usage of limited resources within a defined area. Dried Blood Spots (DBS) are whole blood collected on filter paper and dried. DBS are used for re-testing at a reference laboratory, which is part of Nigeria's External Quality Assessment control in any survey carried out. Testing site results are then compared to reference laboratory results to ensure accuracy of results obtained from the field. DBS require minimal technical skills to collect and do not require cold-chain transportation [3] and therefore have been the most widely studied alternative specimen type [1,4-14]. With improvement in technology, DBS have produced results similar to those of plasma for quantitative viral load analysis [1,15-18] and HIV drug resistance genotyping analysis [4-14,19,20] for both patients on antiretroviral treatment and treatment-naive patients, indicating that DBS could be a viable alternative to plasma for HIV monitoring surveys [19].

Until recently, Whatman 903 (W-903) filter paper was the predominant filter paper used for DBS collection in studies analyzing DNA PCR for early infant diagnosis, HIV-1 loads $[4,16,18]$ and serving as the only filter paper used for HIV drug 
resistance genotyping [4-15,19-21]. Consequently it is also used for routine national HIV prevalence monitoring surveys in Nigeria. With the increasing demand for evidence to guide policy and programming, in resource-constrained countries like Nigeria, DBS have now become the preferred specimen type, thus creating a greater demand for filter paper. Diversifying the type of filter papers available for HIV monitoring surveys could also decrease costs through price competition and increase the availability of filter paper, thus positively impacting low-income countries.

However, most studies that have evaluated DBS filter papers have conducted these studies under controlled laboratory environments with aseptic techniques by highly trained laboratory scientists and stored samples aseptically following international standards [1,14]. While the results of these studies validate the efficacy of using DBS compared to plasma, DBS collection is often done in aseptic techniques, by lower levels of health staff and transported aseptically. The aim of this study was compare the utilities of two DBS collection devices that use the same filter paper, Munktell filter paper (M-TFN) and provide evidence to guide sample collection, storage and transportation in Nigeria. Munktell TFN filter paper meets the Clinical and Laboratory Standards Institute (CLSI) 2007 requirements for blood collection onto filter paper [22].

\section{Methods}

\section{Sample collection}

Seventy two laboratory scientists participated in a three day training exercise which focused on administering both pre- and post-test counselling, collection of specimen from key populations under universal precaution in the field and storage of samples. Twenty samples of each key population target group were sampled and these included female sex workers (brothel and non-brothel), transport workers, armed forces, police and injecting drug users.
Each respondent who consented to HIV counselling and testing was pricked using a retractable lancet. HIV status was ascertained using parallel algorithm according to the national HCT protocol. Two drops of whole blood were also collected on both the conventional DBS card and HemaSpot ${ }^{\mathrm{TM}}$ for quality control using HIV ELISA (Vironostika Ag/Ab). HemaSpot ${ }^{\mathrm{TM}}$ is a tamper resistant, moisture-tight cartridge, which encloses a filter paper and desiccant, thus eliminating contamination of sample and improving sample collection, handling, transport and storage.

Filter paper in HemaSpot ${ }^{\mathrm{TM}}$ is encapsulated in rubber casing with a funnel shaped central opening, which is the only means on introducing blood to the filter paper. The funnel shaped central opening thus allows the controlled and even spread of blood from a central position to the edge of the filter paper. This eliminates uneven drops that may lead to caking and uneven drying which affects sample analysis. The desiccant embedded within the cartridge eliminates the need for prolonged airdrying and the sample is ready to be stored or dispatched within two minutes. Whole blood collected on the conventional DBS card, requires the lab scientist to aim at the centre of each spot while at the same time dropping enough volume of blood and moving quickly over the five-spots designated as dropzone on the card. Subsequently, the card is allowed to air dry for about three hours following manufacturer's guidelines and subsequently stored in a Ziploc bag with desiccants and humidity indicator. All samples were then transported to the reference laboratory for HIV Elisa assay. Ethical approval was provided by the National Health Research Ethics Committee, Nigeria.

\section{HIV rapid test}

HIV rapid test was performed with the parallel national algorithm with the use of Determine (Alere Medical, USA) and

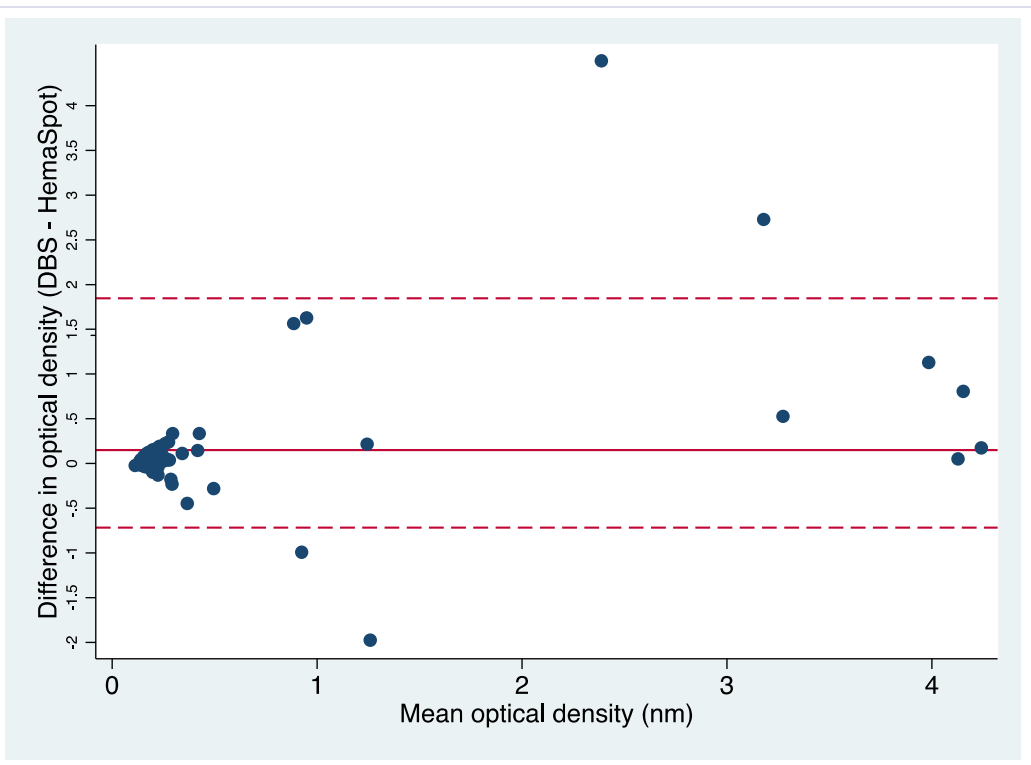

Figure 1: Bland-Altman analysis. 
Unigold (Trinity Biotech, Plc, Bray, Ireland) rapid test kits simultaneously. All discordant tests from the screening were retested using Stat Pak rapid test kit as a tie-breaker. At the time of the rapid testing, the dried blood spots were obtained from all participants on both DBS collection devices.

\section{HIV ELISA assay}

Plain micro tubes were set for sample elution. Wells A1 to E1 were left empty for controls. Blank DBS was punched for negative control. Samples were punched into wells starting at G1 followed by $\mathrm{H} 1$. This was repeated using one blade each for HemaSpot ${ }^{\mathrm{TM}}$. For each punch, card-number was documented on a worksheet. $200 \mu \mathrm{l}$ of PBS buffer was added to each well (except A1 to E1). The plate was tapped from the side three times, for the spots to turn and then the plate was placed on a shaker for $\sim 30$ sec. Wells were covered and placed in fridge overnight to elute (4 degrees Celsius). Testing was done following HIV ELISA DBS protocol developed by Vironostika 4th Generation ELISA (bioMérieux, France).

\section{Results}

\section{Descriptive findings of samples}

A total of ninety-six pairs conventional DBS cards and HemaSpot ${ }^{\mathrm{TM}}$ were collected. Thus a total of ninety-six (96) samples met the ALTC acceptance criteria for samples and were tested in duplicates, yielding a total of 192 specimens. Of the 96 samples accepted for ELISA assay, only 86 samples had rapid test results.

\section{HIV sero-positive samples}

Of the 96 duplicate samples (conventional DBS card and HemaSpot ${ }^{\mathrm{TM}}$ ) assayed, HIV sero-positivity from the conventional DBS card samples was 66.7\% $(n=64)$ while $26 \%(n=25)$ of HemaSpot ${ }^{\mathrm{TM}}$ samples were reactive to HIV. For the rapid test, only $7 \%(\mathrm{n}=6)$ were reactive to HIV.

\section{Concordance between conventional DBS card and HemaSpot $^{\mathrm{TM}}$}

Concordance of results shows the degree of agreement of results between different methods of assay or collection kits. Of the 96 samples accepted for the assay, concordance between conventional DBS card and HemaSpot ${ }^{\mathrm{TM}}$ was $46.9 \%(\mathrm{n}=45)$. Since ELISA is read with optical density of the sample, a test of the difference between the means of all positive and negative concordant samples was also conducted. The Wilcoxon Signrank test was used to estimate the differences between optical densities (OD) of concordant samples. Overall, the mean OD difference between conventional DBS cards (0.571 \pm standard deviation (SD) 1.093) and HemaSpot ${ }^{\mathrm{TM}}(0.42 \pm$ SD 0.839) was significantly different from zero $(p<0.0001)$. The mean OD difference for concordant negative samples between conventional DBS cards (0.164 \pm SD 0.030$)$ and HemaSpot ${ }^{\mathrm{TM}}(0.145$ \pm SD 0.236 ) was significantly different from zero $(p=0.05)$. This finding was also similar for concordant positive samples between conventional DBS cards (0.774 \pm SD 1.295) and HemaSpot ${ }^{\mathrm{TM}}$ (01.205 \pm SD 1.386; $p=0.05)$. Finally the Bland-Altman analysis showed poor agreement between both DBS collection devices with a mean of 0.149 with a standard deviation of 0.641 , which yields a limit of agreement of -0.717 to 1.847 .

\section{Concordance between rapid test, conventional DBS card and HemaSpot ${ }^{\mathrm{TM}}$}

Of the 86 samples available for rapid test, conventional DBS and HemaSpot ${ }^{\mathrm{T}}$, concordance of positive and negative results was evaluated to determine the degree agreeability between rapid test result and conventional DBS card and between rapid test result and HemaSpot ${ }^{\mathrm{TM}}$.

Concordance between rapid test and conventional DBS card was $39.5 \%(n=34)$ while concordance between rapid test and HemaSpot $^{\mathrm{TM}}$ was $75.6 \%(\mathrm{n}=65)$. Of the 34 concordant samples between rapid test and conventional DBS card, 29 samples were negatively concordant while only 5 samples were positively concordant. Of the 65 concordant samples between rapid test and HemaSpot ${ }^{\mathrm{TM}}$, 60 samples were negatively concordant while only 5 samples were positively concordant.

Finally, of the 86 samples, concordance between conventional DBS card and HemaSpot ${ }^{\mathrm{TM}}$ was $50 \%$.

\section{Discussion}

This study highlights the importance of improving sample collection methods. Studies have shown that major issues with laboratory results arise from the pre-analytic phase of analysis, which includes sample collection, and this is applicable to both collection devices [23]. The accuracy of a result can adversely be affected and/or invalidated if the sample is not collected properly, and the spot size varies as a result of (i) co-joined spots where two spots are applied too closely and merge together and (ii) smeared or splattered spots where blood is accidentally smeared before it is completely dried or splattered during spot application $[23,24]$. Sample collection devices designed to eliminate or limit these problems are needed. McMorran et al. (2016) [23] showed higher reliability and consistency of sample transfer to DBS cards when a stabilizing gyroscope was introduced into the sample collection technique.

Overall, the proportion of reactive conventional DBS card samples was very high and raises an alarm on potential issues that may arise in routine HIV surveillance. Contamination between samples during blood collection by the tester or during prolonged air drying of the conventional DBS cards may be accountable for the very high proportion of reactive samples. Another plausible explanation, is that the conventional DBS cards has better physico-chemical properties compared to HemaSpot $^{\mathrm{TM}}$ filter paper and thus has higher bioavailability of blood proteins available for assay. Consequently, this would mean the bioavailability of blood proteins from the HemaSpot ${ }^{\mathrm{TM}}$ is too low to provide valid results from ELISA. However, given that the overall proportion of sero-positive samples from the 2007 and 2010 Integrated Biological and Behavioural Surveillance Surveys [23] were approximately $11 \%$, the results from the HemaSpot ${ }^{\mathrm{TM}}$ present a more conservative possibility of possible upward trend in HIV sero-positivity of key populations in Nigeria. 
The concordance between conventional DBS card and HemaSpot $^{\mathrm{TM}}$ was observed to be very low with poor limit of agreement as evidence by the wide range of optical density using the Bland-Altman analysis. In addition, the low concordance between conventional DBS card and rapid test result, which is the algorithm of the national HCT protocol, suggests that there may be unresolved issues with the skill set of the testers on the field, the rapid test kits used, the ELISA reagent or the type of collection device used. Furthermore the high concordance between the rapid test result and HemaSpot ${ }^{\mathrm{TM}}$ (76\%), compared to the conventional DBS card/rapid test concordance (40\%) suggests that HemaSpot ${ }^{\mathrm{TM}}$ and its lower risk of sample contamination may have contributed to the HemaSpot ${ }^{\mathrm{TM}}$ result being more highly concordant. Lastly, the significant differences in the means of the optical densities of negatively concordant samples, implies that a significant number of key population may have been erroneously misdiagnosed. This has significant implications for HIV prevention as HIV Counseling and Testing (HCT) is a key intervention strategy for effective HIV control in most developing countries including Nigeria. It increases access and knowledge of HIV status, encourages safer sex and is an entry point for HIV care treatment and support services [24].

Given that both devices use the same filter paper, the discordance between the rapid test results and the ELISA results via the different collection devices underscores the urgency of validating the most appropriate collection device for biological samples for intended assays. Furthermore, as manufacturers of ELISA kits are moving away from third generation ELISA kits that assay only antibodies, it is imperative that the Nigerian surveillance team align its quality control protocol to current international standards. Fourth generation ELISA kits, have the ability to detect both HIV antigens and antibodies to HIV and thus provide added information on new infections in Nigeria, while providing a more accurate result of prevalence of the disease in question.

\section{Limitation}

This study has some limitations. The ELISA assay used for the pilot was a fourth generation ELISA kit capable of detecting both HIV antigens and antibodies to HIV. This may be a cause of the discordance in addition to contamination. However, given that HIV incidence is generally low; it is unlikely that the higher reactive samples from the conventional DBS card were as a result of new infections only. The study also did not conduct genotyping of HIV DNA to determine if contamination is a significant contributor to the higher reactivity of the conventional DBS card and HemaSpot ${ }^{\mathrm{TM}}$ compared to the rapid test. Finally, serum/plasma of dried blood spot samples was not available for comparison with results from conventional DBS card collection devices. However, while it is acknowledged that this is the gold standard, its unavailability does not invalidate these results and thus pertinent issues on sample collection among lower cadre of health staff in resource limited settings have been brought to the fore.

\section{Conclusion}

This study has highlighted significant issues in the sample collection and analytic processes of the HIV surveillance. It is unlikely that two different devices will yield the same result, however given that they both use the same filter paper; we would expect a high level of agreement between the devices. Overall sero-positivity of rapid test, conventional DBS card and HemaSpot $^{\mathrm{TM}}$ was $7 \%, 67 \%$ and $29 \%$ respectively and concordance between rapid test and conventional DBS card was $39 \%$ while it was $76 \%$ for rapid test and $\mathrm{HemaSpot}^{\mathrm{TM}}$. It is unlikely that the increase in sero-positivity in conventional DBS card and HemaSpot is exclusive to new infections identified by the antigenic capability of the ELISA kit. Furthermore, given that both collection devices used the same filter paper, it underscores the urgency of validating the most appropriate collection device for biological samples for intended assays. The limitation in the scope of the study did not provide the avenue to conduct further analysis to identify the cause of the discordance. However, the high level of discordance of results, especially between rapid test and conventional DBS card raises a cause for alarm and necessitates a more rigorous quality control for the field based sample collection. In addition, urgent research is needed to optimize ELISA kits with appropriate dried blood spot collection devices in order to ensure that results from the quality control of surveillance studies achieve their aim and purpose of validating the results of the national algorithm.

\section{References}

1. Rottinghaus E, Bile E, Modukanele M, Maruping M, Mine M, et al. Comparison of Alhstrom Grade 226, Munktell TFN, and Whatman 903 Filter Papers for Dried Blood Spot Specimen Collection and Subsequent HIV-1 Load and Drug Resistance Genotyping Analysis. J Clin Microbiol. 2013;51(1):55-60. doi: 10.1128/JCM.02002-12.

2. Bennett DE, Bertagnolio S, Sutherland D, Gilks CF. The World Health Organization's global strategy for prevention and assessment of HIV drug resistance. Antivir Ther. 2008;13 Suppl 2: 1-13.

3. Hamers RL, Smit PW, Stevens W, Schuurman R, Rinke de Wit TF. Dried fluid spots for HIV type-1 viral load and resistance genotyping:a systematic review. Antivir Ther. 14:619-629.

4. Bertagnolio S, Soto-Ramirez L, Pilon R, Rodriguez R, Viveros M, Fuentes L, et al. HIV-1 drug resistance surveillance using dried whole blood spots. Antivir Ther. 2007;12(1):107-13.

5. Buckton AJ, Bissett SL, Myers RE, Beddows S, Edwards S, Cane PA, et al. Development and optimization of an internally controlled dried blood spot assay for surveillance of human immunodeficiency virus type-1 drug resistance. J Antimicrob Chemother. 2008;62(6):11918. doi: $10.1093 / \mathrm{jac} / \mathrm{dkn} 412$.

6. Garrido C, Zahonero N, Fernandes D, Serrano D, Silva AR, Ferraria N, et al. Subtype variability, virological response and drug resistance assessed on dried blood spots collected from HIV patients on antiretroviral therapy in Angola. J Antimicrob Chemother. 2008;61(3):694-8. doi: 10.1093/jac/dkm515.

7. Hallack R, Doherty LE, Wethers JA, Parker MM. Evaluation of dried blood spot specimens for HIV-1 drug-resistance testing using the Trugene HIV-1 genotyping assay. J Clin Virol. 2008;41(4):283-7. doi: 10.1016/j.jcv.2007.12.011.

8. Lira R, Valdez-Salazar H, Vazquez-Rosales G, Rojas-Montes O, Ruiz- 
Tachiquin M, Torres-Ibarra R, et al. Genotypic testing for HIV-1 drug resistance using dried blood samples. Arch Virol. 2010;155(7):1117-25. doi: 10.1007/s00705-010-0696-y.

9. Masciotra S, Garrido C, Youngpairoj AS, McNulty A, Zahonero N, Corral A, et al. High concordance between HIV-1 drug resistance genotypes generated from plasma and dried blood spots in antiretroviralexperienced patients. AIDS. 2007;21:2503-2511.

10. Steegen K, Luchters S, Demecheleer E, Dauwe K, Mandaliya K, Jaoko W, et al. Feasibility of detecting human immunodeficiency virus type 1 drug resistance in DNA extracted from whole blood or dried blood spots. J Clin Microbiol. 2007;45(10):3342-51.

11.Yang C, McNulty A, Diallo K, Zhang J, Titanji B, Kassim S, et al. Development and application of a broadly sensitive dried-blood-spotbased genotyping assay for global surveillance of HIV-1 drug resistance. J Clin Microbiol. 2010;48(9):3158-64. doi: 10.1128/JCM.00564-10.

12. Youngpairoj AS, Masciotra S, Garrido C, Zahonero N, de Mendoza C, Garcia-Lerma JG, et al. HIV-1 drug resistance genotyping from dried blood spots stored for 1 year at 4 degrees C. J Antimicrob Chemother. 2008;61(6):1217-1220. doi: 10.1093/jac/dkn100.

13.Ziemniak C, George-Agwu A, Moss WJ, Ray SC, Persaud D. Asensitive genotyping assay for detection of drug resistance mutations in reverse transcriptase of HIV-1 subtypes B and C in samples stored as dried blood spots or frozen RNA extracts. J Virol Methods. 2006;136(1-2):238-47.

14. Johannessen A, Garrido C, Zahonero N, Sandvik L, Naman E, Kivuyo SL, et al. Dried blood spots perform well in viral load monitoring of patients who receive antiretroviral treatment in rural Tanzania. Clin Infect Dis. 2009;49(6):976-81. doi: 10.1086/605502.

15. Monleau M, Butel C, Delaporte E, Boillot F, Peeters M. Effect of storage conditions of dried plasma and blood spots on HIV-1 RNA quantification and PCR amplification for drug resistance genotyping. J Antimicrob Chemother. 2010 ;65(8):1562-6. doi: 10.1093/jac/dkq205.

16. Van Deursen P, Oosterlaken T, Andre P, Verhoeven A, Bertens L, Trabaud MA,et al. Measuring human immunodeficiency virus type 1 RNA loads in dried blood spot specimens using NucliSENS EasyQ HIV-1 v2.0. J Clin Virol. 2010;47(2):120-5. doi: 10.1016/j.jcv.2009.11.021.

17. Rottinghaus EK, Ugbena R, Diallo K, Bassey O, Azeez A, Devos J, et al. Dried blood spot specimens are a suitable alternative sample type for HIV-1 viral load measurement and drug resistance genotyping in patients receiving firstline antiretroviral therapy. Clin Infect Dis. 2012;54(8):1187-95. doi: 10.1093/cid/cis015.

18.Zhou Z, Wagar N, DeVos JR, Rottinghaus E, Diallo K, Nguyen DB, Bassey, et al. Optimization of a low cost and broadly sensitive genotyping assay for HIV-1 drug resistance surveillance and monitoring in resource-limited settings. PLoS One 2011;6:e28184. doi:10.1371/journal.pone.0028184.

19. Charpentier C, Gody JC, Tisserand P, Matta M, Fournier J, Mbitikon 0 , et al. Usefulness of a genotypic resistance test using dried blood spot specimens in African HIV-infected children with virological failure according to the 2010-revised WHO criteria. Arch Virol. 2011;156(9):1603-6. doi: 10.1007/s00705-011-0997-9.

20. Menzies N, Abang B, Wanyenze R, Nuwaha F, Mugisha B, Coutinho A, et al. The costs and effectiveness of four HIV counseling and testing strategies in Uganda. AIDS 2009;23:395-401.

21. CLSI. 2007. Approval standard. CLSI publication number LA4-A5. Wayne, PA.

22. Federal Ministry of Health. 2007. Integrated Biological and Behavioural Surveillance Survey. Federal Ministry of Health, Abuja.

23. McMorran D, Chung DC, Toth M, Liew OW, Muradoglu M, Ng TW. Stabilized dried blood spot collection. Anal Biochem. 2016;506:2830. doi:10.1016/j.ab.2016.04.015.

24. Burnett JEC, Dried blood spot sampling: practical considerations and recommendation for use with preclinical studies. Bioanalysis. 2011;3(10):1099-107.doi:10.4155/BI0.11.68. 